\title{
ON THE ERGODIC THEOREM WITHOUT ASSUMPTION OF POSITIVITY
}

\author{
BY R. V. CHACON \\ Communicated by John C. Oxtoby, December 1, 1960
}

0. Introduction. Dunford and Schwartz $[3 ; 5]$ have recently obtained a generalization of an ergodic theorem of E. Hopf [4] to include nonpositive operators in spaces of complex valued functions. Their method of proof is to majorize the operator in question by a positive one, and then to apply Hopf's results. We give a direct proof of a maximal ergodic lemma for operators which are not necessarily positive, in spaces of functions which take their values in an arbitrary Banach space. This lemma is used to further generalize Hopf's theorem so that it applies to operators which are not necessarily positive, in spaces of functions which take their values in a reflexive Banach space. This application of the lemma uses the Kakutani mean ergodic theorem. The method of proof we have used appears to be shorter and simpler than the methods given previously for more special results. Further, our result has the additional advantage that it is sufficiently general to give as a direct corollary a theorem of Kakutani and of Beck and Schwartz [1] (stated as Theorem 2 in this paper).

1. Main results. Let $\mathfrak{X}$ be a Banach space and $(S, \Sigma, \mu)$ a $\sigma$-finite measure space. Let $L_{p}(S, \Sigma, \mu, \mathfrak{X}), 1 \leqq p<+\infty$, herein called simply $L_{p}(S, \mathfrak{X})$ denote the space of all strongly measurable $\mathfrak{X}$-valued functions $f$ defined on $S$ for which the norm given by

$$
\|f\|_{p}=\left(\int_{S}\|f(s)\|^{p}(d s)\right)^{1 / p}<+\infty ;
$$

and let $L_{\infty}(S, \Sigma, \mu, \mathfrak{X})$, herein called simply $L_{\infty}(S, \mathfrak{X})$, denote the space of all strongly measurable $\mathfrak{X}$-valued functions $f$ defined on $S$ for which

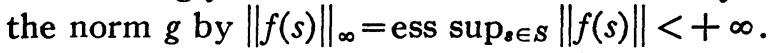

Let $T$ be a linear operator in $L_{1}(S, \mathfrak{X})$ such that $\|T\|_{1} \leqq 1$, where $\|T\|_{1}=\sup _{\|f\|_{1} \leq 1}\|T f\|_{1}$, and such that $\|T f\|_{\infty} \leqq\|f\|_{\infty}$ for any function $f$ in $L_{1} \cap L_{\infty}$.

Extend $T$, if necessary, so that it is defined on each $L_{p}, 1 \leqq p<+\infty$. When convenient we shall suppress the argument of a function, writing $f$ for $f(s)$. Whenever a division by zero is indicated the term in which this appears will be taken to be zero.

If $f$ is a strongly measurable function on $S$ and $a>0$, let 


$$
\begin{aligned}
& f^{a-}(s)=\{\min (a,\|f(s)\|)\} \cdot \frac{f(s)}{\|f(s)\|}, \\
& f^{a+}(s)=\{\max (a,\|f(s)\|)-a\} \cdot \frac{f(s)}{\|f(s)\|},
\end{aligned}
$$

and

$$
e^{*}(a)=\left\{s: \sup _{n \geqq 0}\left\|\frac{1}{n+1} \sum_{k=0}^{n} T^{k} f(s)\right\|>a\right\} .
$$

Note that, according to our convention, $f^{a+}(s)$ and $f^{a-}(s)$ are zero whenever $f(s)$ is.

2. Maximal ergodic lemma. If $f \in L_{p}(S, \mathfrak{X}), 1 \leqq p<\infty$, then

$$
\int_{e^{*}(a)}\left(a-\left\|f^{a-}(s)\right\|\right) d s \leqq \int\left\|f^{a+}(s)\right\| d s .
$$

Proof. Define inductively

$$
\begin{aligned}
f_{0}(s)= & f^{a+}(s) \\
f_{j+1}(s)= & T f_{j}(s)-\frac{T f_{j}(s)}{\left\|T f_{j}(s)\right\|} \\
& \cdot \min \left\{\left\|T f_{j}(s)\right\|, a-\left\|f^{a-}(s)\right\|-\sum_{k=0}^{j-1}\left\|T f_{k}(s)-f_{k+1}(s)\right\|\right\}, \\
d_{0}(s)= & 0, \\
d_{j+1}(s)= & T f_{j}(s)-f_{j+1}(s) .
\end{aligned}
$$

These definitions imply

$$
T^{j} f=T^{j} f^{a-}+f_{j}+\sum_{k=0}^{j} T^{j-k} d_{k}
$$

since the assertion is trivial for $j=0$ and if we assume it for $j=n$ then it follows that

$$
\begin{aligned}
T^{n+1} f & =T^{n+1} f^{a-}+T f_{n}+\sum_{k=0}^{n} T^{n+1-k} d_{k} \\
& =T^{n+1} f^{a-}+\sum_{k=0}^{n+1} T^{n+1-k} d_{k}+T f_{n}-d_{n+1} \\
& =T^{n+1} f^{a}-f_{n+1}+\sum_{k=0}^{n+1} T^{n+1-k} d_{k} .
\end{aligned}
$$


The definitions also imply that for each $j$

$$
\left\|f^{a-}\right\|+\sum_{k=0}^{j}\left\|d_{k}\right\| \leqq a,
$$

since it follows from (2.1) by induction that

$$
\left\|d_{j}(s)\right\|=\min \left\{\left\|T f_{j-1}(s)\right\|, a-\left\|f^{a-}(s)\right\|-\sum_{k=0}^{j-1}\left\|d_{k}(s)\right\|\right\}
$$

and this implies that

$$
\left\|d_{j}(s)\right\| \leqq a-\left\|f^{a-}(s)\right\|-\sum_{k=0}^{j-1}\left\|d_{k}(s)\right\| .
$$

Now from (2.2) we see that

$$
\begin{aligned}
\sum_{j=0}^{n} T^{i f} & =\sum_{j=0}^{n} T^{i f a-}+\sum_{j=0}^{n} f_{j}+\sum_{j=0}^{n} \sum_{k=0}^{g} T^{j-k} d_{k} \\
& =\sum_{j=0}^{n} T^{j}\left(f^{a-}+\sum_{k=0}^{n-j} d_{k}\right)+\sum_{j=0}^{n} f_{j},
\end{aligned}
$$

which implies that

$$
\begin{aligned}
\left\|\sum_{j=0}^{n} T^{j} f\right\| \leqq & \left\|\sum_{j=1}^{n} T^{i}\left(f^{a-}+\sum_{k=0}^{n-j} d_{k}\right)\right\|+\left\|f^{a-}\right\| \\
& +\sum_{k=0}^{n}\left\|d_{k}\right\|+\sum_{j=0}^{n}\left\|f_{j}\right\| .
\end{aligned}
$$

From equation (2.4) and (2.3) and the fact that $T_{j}, j=1, \cdots, n$ cannot increase the essential supremum we obtain

$$
\left\|\sum_{j=0}^{n} T^{j} f\right\| \leqq\left\|f^{a}-\right\|+\sum_{k=0}^{n}\left\|d_{k}\right\|+n a+\sum_{j=0}^{n}\left\|f_{j}\right\|,
$$

from which it follows that

$$
a \leqq\left\|f^{a}-\right\|+\sum_{k=0}^{\infty}\left\|d_{k}\right\|+\sum_{k=0}^{\infty}\left\|f_{k}\right\|
$$

on $e^{*}(a)$, and thus that

$$
a \leqq\left\|f^{a}-\right\|+\sum_{k=0}^{\infty}\left\|d_{k}\right\|
$$

at least on the subset of $e^{*}(a)$ where $\sum_{k=0}^{\infty}\left\|f_{k}\right\|=0$. To see that (2.5) 
also holds a.e. on that part of $e^{*}(a)$ where $\sum_{k=0}^{\infty}\left\|f_{k}\right\|>0$, note that if we let $e_{j}^{*}(a)$ be the set where $\left\|f_{j}(s)\right\|>0$, then on $e_{j}^{*}(a)$ we have

$$
a \leqq\left\|f^{a}-\right\|+\sum_{k=0}^{i}\left\|d_{k}\right\|,
$$

which implies (2.5) on $e_{j}^{*}(a)$. Now (2.3) and (2.5) imply that $a=\left\|f^{a-}\right\|$ $+\sum_{k=0}^{\infty}\left\|d_{k}\right\|$ a.e. on $e^{*}(a)$. It follows that

$$
\int \sum_{k=0}^{\infty}\left\|d_{k}\right\| d s=\int_{e *(a)}\left(a-\left\|f^{a-}(s)\right\|\right) d s .
$$

From (2.1) we see that $\left\|T f_{k-1}\right\|=\left\|d_{k}\right\|+\left\|f_{k}\right\|$, and since $\|T\|_{1} \leqq 1$, that

$$
\int\left\|f_{k-1}(s)\right\| d s \geqq \int\left\|d_{k}(s)\right\| d s+\int\left\|f_{k}(s)\right\| d s,
$$

and thus that

$$
\int\left\|f^{a+}(s)\right\| d s \geqq \int \sum_{k=0}^{\infty}\left\|d_{k}(s)\right\| d s .
$$

This equation together with (2.6) proves the lemma.

Theorem 1. If $\mathfrak{X}$ is reflexive and $1 \leqq p<\infty$ and $f \in L_{p}(S, \mathfrak{X})$, the limit

$$
\lim _{n \rightarrow \infty} 1 / n \sum_{m=0}^{n-1} T^{m} f(s)
$$

exists strongly for almost all $s \in S$. Furthermore, if $1<p<+\infty$ then there exists a function $f^{*} \in L_{p}$ such that

$$
\left\|1 / n \sum_{m=0}^{n-1} T^{m} f(s)\right\| \leqq\left\|f^{*}(s)\right\|, \quad \text { for almost all } s \in S .
$$

Proof. The maximal ergodic lemma implies that if $\left\{g_{k}\right\}$ is a sequence of functions in $L_{p}(S, \mathfrak{X}), p \geqq 1$ such that

$$
\lim _{x \rightarrow \infty} \int\left\|g_{k}^{a+}(s)\right\| d s=0
$$

for each $a>0$, then for each $b>0$ the set where

$$
\sup _{n \geqq 1}\left\|\frac{g_{k}+\cdots+T^{n-1} g_{k}}{n}\right\|>b
$$

has measure which tends to zero as $k \rightarrow \infty$. We may now get the first 
part of the theorem by noting that we may, using the Kakutani mean ergodic theorem (which we may apply by the Riesz convexity theorem, since $\mathfrak{X}$ is supposed to be reflexive) approximate $f$ by a sequence of functions $\left\{h_{k}\right\}$ in the sense that $g_{k}=f-h_{k}$ satisfies (2.7) and such that for each $k$,

$$
\lim _{n \rightarrow \infty} \frac{h_{k}+\cdots+T^{n-1} h_{k}}{n}
$$

exists almost everywhere.

The second part of the theorem follows from our Lemma 1 as in the proof given by Dunford and Schwartz.

We now state and sketch the proof of the ergodic theorem of Kakutani and of Beck and Schwartz mentioned in the introduction.

THEOREM 2. Let $\mathfrak{X}$ be reflexive and let there be defined on $S$ a strongly measurable function $U$, with values in the $B$-space $B(\mathfrak{X})$ of bounded linear operators on $\mathfrak{X}$. Suppose that $\left\|U_{s}\right\| \leqq 1$ for all $s \in S$. Let $q$ be a measure preserving transformation in $(S, \Sigma, \mu)$. Then for each $f \in L_{1}(S, \mathfrak{X})$ there is an $\bar{f} \in L_{1}(S, \mathfrak{X})$ such that the strong limit

$$
\lim _{n \rightarrow \infty} n^{-1} \sum_{i=1}^{n} U_{s} U_{q(s)} \cdots U_{q^{i-1}(s)} \cdot f\left(q^{i}(s)\right)=\bar{f}(s)
$$

almost everywhere on $S$ ( $q^{i}$ denotes the ith iterate of $q$ ).

Proof. Define

$$
T f(s)=U_{s} f(q(s)) .
$$

It can be seen that $T$ satisfies the conditions of Theorem 1 and thus the conclusion follows at once.

\section{REFERENCES}

1. A. Beck and J. T. Schwartz, A vector valued random ergodic theorem, Proc. Amer. Math. Soc. vol. 8 (1957) pp. 1049-1059.

2. R. V. Chacon and D. S. Ornstein, A general ergodic theorem, Illinois J. Math. vol. 4 (1960) pp. 153-160.

3. N. Dunford and J. T. Schwartz, Convergence almost everywhere of operator averages, J. Math. Mech. vol. 5 (1956) pp. 129-178.

4. E. Hopf, The general temporally discrete Markoff process, J. Math. Mech. vol. 3 (1954) pp. 13-45.

5. J. T. Schwartz, Another proof of E. Hopf's ergodic lemma, Comm. Pure Appl. Math. vol. 12 (1959) pp. 399-401.

UNIVERSITY OF WISCONSIN 